\title{
ON BORDISM GROUPS OF IMMERSIONS
}

BY

GUILLERMO PASTOR

\begin{abstract}
The bordism group of immersions of oriented $n$-manifolds into $\mathbf{R}^{n+k}$ is identified with the stable homotopy group $\Pi_{n+k}^{s}(\operatorname{MSO}(k))$. We study these groups for $n-2 \leqslant k \leqslant n$, and discuss the behaviour of double points and their relation with the corresponding bordism groups of embeddings.
\end{abstract}

1. Introduction. Let $I \Omega_{n, k}$ denote the bordism group of immersions of oriented $n$-manifolds into $\mathbf{R}^{n+k}$. Here a bordism between two immersions $i_{0}: M_{0} \hookrightarrow \mathbf{R}^{n+k}$ and $i_{1}: M_{1} \hookrightarrow \mathbf{R}^{n+k}$ is an immersion of a compact oriented $(n+1)$-manifold $j: W \hookrightarrow$ $\mathbf{R}^{n+k} \times I$ such that $\partial W=M_{0} \cup-M_{1}$ and $\left.j\right|_{M_{0}}=i_{0} \times\{0\}$ and $\left.j\right|_{M_{1}}=i_{1} \times\{1\}$. In the usual manner bordism defines an equivalence relation and bordism classes form an abelian group (under disjoint union) which is identified with the stable homotopy group $\pi_{n+k}^{s}(\mathrm{MSO}(k))$, of the Thom space $\mathrm{MSO}(k)$ of the canonical oriented $k$-plane bundle over $\mathrm{BSO}(k)$.

The object of this paper is to study the groups $I \Omega_{n, k}$ for $n-2 \leqslant k \leqslant n$ and to discuss the behaviour of double points and the relation of these groups with the corresponding bordism groups of embeddings.

Bordism groups of immersions were studied first by Wells [We] who determined the unoriented groups $I \Re_{n, n}$ and $I \Re_{4 n, 4 n-1}$. These results were extended by Koschorke and Olk who completed the computations of $I \mathfrak{N}_{n, k}$ for $n-2 \leqslant k \leqslant n$ (see $[\mathbf{K}, \S 10])$. We shall make use of these computations..

This work is part of the author's thesis under the supervision of B. J. Sanderson at Warwick University. It is a pleasure to express thanks for his help and for helpful conversations with the topology staff at the Centro de Investigación del IPN.

2. Some exact sequences involving bordism groups of immersions. We describe three exact sequences and compute some low-dimensional bordism groups appearing in them. The first sequence was obtained by Szücs [Sz] and Koschorke [K]. The other two sequences are due to Salomonsen [Sa]. We refer to these articles for a detailed description of the sequences.

Given a subgroup $G$ of the orthogonal group $O(m)$ we will denote by $\Omega_{j}^{G}$ the bordism group of $j$-manifolds whose stable normal bundle admits a reduction to $G$. We will be mainly interested in the cases where $G$ is

$$
z(k)=\left\{\left(\begin{array}{cc}
A & 0 \\
0 & A
\end{array}\right),\left(\begin{array}{cc}
0 & A \\
A & 0
\end{array}\right): A \in \mathrm{SO}(k)\right\},
$$

Received by the editors May 31, 1983.

1980 Mathematics Subject Classification. Primary 57R90, 57R75; Secondary 55Q52.

(1984 American Mathematical Society $0025-5726 / 84 \$ 1.00+\$ .25$ per page 
or

$$
w(k)=\left\{\left(\begin{array}{cc}
A & 0 \\
0 & B
\end{array}\right),\left(\begin{array}{cc}
0 & A \\
B & 0
\end{array}\right): A, B \in \mathrm{SO}(k)\right\}
$$

$$
\Delta \mathrm{SO}=\left\{\left(\begin{array}{cc}
A & 0 \\
0 & A
\end{array}\right): A \in \mathrm{SO}(l), l \gg j\right\} .
$$

Let $f: I \Omega_{n, k} \rightarrow \Omega_{n}$ denote the forgetful homomorphism that retains the oriented bordism class of the domain of a class of immersions and let $E \Omega_{n . k}$ stand for the bordism group of classes of embeddings of oriented $n$-manifolds in $\mathbf{R}^{n+k}$. From now on we will assume $n<2 k-1$. This is the metastable range and corresponds to the range in which only double points arise from self-transverse immersions.

2.1 Proposition. Let $n<2 k-1$. Then the following sequences are exact:

$$
\begin{gathered}
\cdots \rightarrow \Omega_{n-k}^{z(k)} \stackrel{\partial}{\rightarrow} I \Omega_{n, k} \stackrel{f}{\rightarrow} \Omega_{n} \rightarrow \Omega_{n-k-1}^{z(k)} \rightarrow \cdots, \\
\cdots \rightarrow \Omega_{n-k}^{\Delta \mathrm{SO}} \rightarrow I \Omega_{n, k} \stackrel{g}{\rightarrow} I \Omega_{n, k+1} \stackrel{e}{\rightarrow} \Omega_{n-k-1}^{\Delta \mathrm{SO}} \rightarrow \cdots, \\
\cdots \rightarrow \Omega_{n-k+1}^{w(k)} \rightarrow E \Omega_{n, k} \stackrel{h}{\rightarrow} I \Omega_{n, k} \stackrel{D}{\rightarrow} \Omega_{n-k}^{n(k)} \rightarrow \cdots,
\end{gathered}
$$

Here $g, h$ are the obvious forgetful homomorphisms. Let $j_{k}: S^{k} \hookrightarrow \mathbf{R}^{2 k}$ be defined by $j_{k}\left(t, u_{1}, \ldots, u_{k}\right)=\left((t+1) u_{1}, \ldots,(t+1) u_{k},(1-t) u_{1}, \ldots,(1-t) u_{k}\right)$, where $S^{k}$ is the unit sphere in $\mathbf{R}^{k+1}$ with coordinates $\left(t, u_{1}, \ldots, u_{k}\right)$. Note that $j_{k}$ is an immersion with precisely one double point and that $j_{k}\left(S^{k}\right)$ is $z(k)$-invariant. If $[N]$ represents an arbitrary class in $\Omega_{n-k}^{z(k)}$ then associated to a tubular neighbourhood of an embedding $N \subset \mathbf{R}^{n+k}$ there is a fibre bundle with fibre $j_{k}\left(S^{k}\right)$. The total space of this bundle represents $\partial[N]$.

The homomorphism $e: I \Omega_{n, k+1} \rightarrow \Omega_{n-k-1}^{\Delta \mathrm{SO}}$ is defined as follows. Choose a representative immersion $M^{n} \hookrightarrow \mathbf{R}^{n+k+1}$ with normal bundle $v$. Consider $M$ embedded in $v$ via the zero section and take a section $s: M \rightarrow v$ which is transverse to $M$. $e\left(\left[M \hookrightarrow R^{n+k+1}\right]\right)$ is represented by the intersection manifold $M \cap s(M)$.

If $N^{n} \stackrel{i}{\hookrightarrow} R^{n+k}$ is a self-transverse immersion of an oriented manifold $N$, then $D[i]$ is represented by the double-points manifold.

The other homomorphisms appearing in these sequences can also be defined in geometric terms (see the references above).

Koschorke [K, 9.3] has developed a long exact sequence which is useful in computing low-dimensional bordism groups. The groups $\Omega_{i}^{z(k)}, \Omega_{i}^{\Delta \mathrm{SO}}, \Omega_{i}^{w(k)}, 0 \leqslant i \leqslant$ 2 , can be computed using this sequence.

2.5 Proposition. The bordism groups $\Omega_{i}^{z(k)}, 0 \leqslant i \leqslant 2, k>2$, are given by the following table:

\begin{tabular}{||c|c|c|c||}
\multicolumn{1}{c}{} & $i=0$ & $i=1$ & $i=2$ \\
\hline$k \equiv 1(4)$ & $\mathbf{Z}_{2}$ & $\mathbf{Z}_{2}$ & $\mathbf{Z}_{2} \oplus \mathbf{Z}_{8}$ \\
\hline$k \equiv 2(4)$ & $\mathbf{Z}$ & $\mathbf{Z}_{4}$ & $\mathbf{Z}_{2}$ \\
\hline$k \equiv 3(4)$ & $\mathbf{Z}_{2}$ & 0 & $\mathbf{Z}_{2} \oplus \mathbf{Z}_{2}$ \\
\hline$k \equiv 0(4)$ & $\mathbf{Z}$ & $\mathbf{Z}_{2} \oplus \mathbf{Z}_{2}$ & $\mathbf{Z}_{2} \oplus \mathbf{Z}_{2} \oplus \mathbf{Z}_{2}$ \\
\hline
\end{tabular}


2.6 Proposition. The bordism groups $\Omega_{i}^{w(k)}, 0 \leqslant i \leqslant 2, k>2$, are given by the table:

\begin{tabular}{||c|c|c|c||}
\multicolumn{1}{c}{} & $i=0$ & $i=1$ & $i=2$ \\
\hline$k \equiv 1(4)$ & $\mathbf{Z}_{2}$ & 0 & $\mathbf{Z}_{4}$ \\
\hline$k \equiv 2(4)$ & $\mathbf{Z}$ & $\mathbf{Z}_{2}$ & 0 \\
\hline$k \equiv 3(4)$ & $\mathbf{Z}_{2}$ & 0 & $\mathbf{Z}_{2} \oplus \mathbf{Z}_{2}$ \\
\hline$k \equiv 0(4)$ & $\mathbf{Z}$ & $\mathbf{Z}_{2}$ & $\mathbf{Z}_{2}$ \\
\hline
\end{tabular}

2.7 Proposition. The groups $\Omega_{i}^{\Delta \mathrm{SO}}$ are isomorphic to $\mathbf{Z}, \mathbf{Z}_{2}$ and $\mathbf{Z}_{2} \oplus \mathbf{Z}_{2}$ for $i=0,1,2$, respectively.

3. Bordism groups of immersions. We now study sequences (2.2) and (2.3). The groups $I \Omega_{n, k}(n-2 \leqslant k \leqslant n)$ are determined except for extension problems in some cases. The unoriented version of sequence (2.2) was studied by Koschorke [K]. The extension problems which arise from (2.2) are, in general, more difficult to solve than in the unoriented case (see [K, 10.4]). The unoriented analogue of (2.3), can be deduced from results of [ $\mathbf{K}$ and $\mathbf{S a}$ ]. We will make use of these sequences to solve some extension problems. In particular, a detailed description of some of the homomorphisms between the bordism groups $\Omega_{i}^{z(k)}, \Omega_{i}^{\Delta \text { SO }}$ and the unoriented analogues will be needed. This can be achieved by comparing the corresponding long exact sequences of [K, 9.3].

3.1 Theorem. For $n>0, I \Omega_{n, n} \cong \Omega_{n} \oplus \mathbf{Z}$ if $n$ is even and $I \Omega_{n, n} \cong \Omega_{n} \oplus \mathbf{Z}_{2}$ if $n$ is odd. The $\mathbf{Z}$ or $\mathbf{Z}_{2}$ factor is generated by the class of the immersion $j_{n}: S^{n} \hookrightarrow \mathbf{R}^{2 n}$.

Proof. From the Whitney immersion theorem and sequence (2.2) we get the commutative diagram with horizontal exact sequence:

$$
\begin{aligned}
0 \rightarrow \quad \Omega_{0}^{z(k)} & \rightarrow I \Omega_{n, n} \rightarrow \Omega_{n} \rightarrow 0 \\
& \cong \searrow D \\
& \Omega_{0}^{w(k)}
\end{aligned}
$$

3.2 THEOREM. For $n>3$ the groups $I \Omega_{n, n-1}$ are given by

$$
I \Omega_{n, n-1} \cong \begin{cases}\Omega_{n} & \text { for } n \equiv 0(4), \\ \Omega_{n} \oplus \mathbf{Z}_{2} & \text { for } n \equiv 2(4) \text { or } n+1 \text { a power of } 2, \\ \Omega_{n} \oplus \mathbf{Z}_{4} & \text { for } n \equiv 3(4), n+1 \text { not a power of } 2 .\end{cases}
$$

If $n \equiv 1$ (4) then $I \Omega_{n, n-1}$ is an extension of $\Omega_{n} \oplus \mathbf{Z}_{2}$ by $\mathbf{Z}_{2}$.

Proof. If $n+1$ is not a power of 2 then every orientable $(n+1)$-manifold immerses in $\mathbf{R}^{2 n}$ [Mah-P]. Thus sequence (2.2) takes the form

$$
0 \rightarrow \Omega_{1}^{z(n-1)} \rightarrow I \Omega_{n, n-1} \rightarrow \Omega_{n} \rightarrow 0 .
$$

The case $n \equiv 0$ follows immediately from 2.5. The splitting of this sequence for $n \equiv 2$ (4) follows by comparison with the corresponding unoriented sequence. 
If $n \equiv 1$ or 3 (4) then $\left|\Omega_{1}^{z(n-1)}\right|=4$ and hence $\left|I \Omega_{n, n-1}\right|=4\left|\Omega_{n}\right|$, provided $n+1$ is not a power of 2 . Sequence (2.3) reduces then to

$$
0 \rightarrow \mathbf{Z}_{2} \rightarrow I \Omega_{n, n-1} \rightarrow \Omega_{n} \oplus \mathbf{Z}_{2} \rightarrow 0 .
$$

If $n \equiv 3$ (4) this extension is nontrivial, as every element in $\Omega_{n}$ has order 2 and $\mathbf{Z}_{4} \cong \Omega_{1}^{z(n-1)}$ injects into $I \Omega_{n, n-1}$.

Finally assume $n+1$ is a power of 2. By [Mah-P, 4.2.1] $\mathbf{C} P^{(n+1) / 2}$ does not immerse up to cobordism in $\mathbf{R}^{2 n}$. Hence $\Omega_{1}^{z(n-1)}$ does not inject into $I \Omega_{n, n-1}$ and sequence (2.3) becomes

$$
\mathbf{Z}_{2} \stackrel{0}{\rightarrow} I \Omega_{n, n-1} \rightarrow \Omega_{n} \oplus \mathbf{Z}_{2} \rightarrow 0 .
$$

We now study the groups $I \Omega_{n, n-2}, n>5$. Let $\alpha(k)$ denote the number of ones in the binary expansion of an integer $k$.

If $\alpha(n+1)>2$ then the forgetful homomorphism $I \Omega_{n+1, n-2} \rightarrow \Omega_{n+1}$ is onto. This follows either by Cohen's immersion theorem $[\mathbf{C}]$ or by showing that each $(n+1)$ dimensional multiplicative generator has a representative that immerses in $\mathbf{R}^{2 n-1}$ (see [Wa, 0]). If $n+1 \equiv 2$ (4) then $I \Omega_{n+1, n-2} \rightarrow \Omega_{n+1}$ is always onto, as there is a system of generators of $\Omega_{*}$ with no elements in these dimensions [Wa]. Hence if either $\alpha(n+1)>2$ or $n+1 \equiv 2$ (4) then we get an exact sequence

$$
0 \rightarrow \Omega_{2}^{z(n-2)} \rightarrow I \Omega_{n, n-2} \rightarrow \Omega_{n} \rightarrow 0 .
$$

3.3 THEOREM. Let $n>5$.

(i) If $n \equiv 0$ (4) then $I \Omega_{n, n-2} \cong \Omega_{n} \oplus \mathbf{Z}_{2}$ if $\alpha(n)>1$. If $\alpha(n)=1$ then $I \Omega_{n, n-2}$ is isomorphic to the subgroup of $\Omega_{n}$ consisting of classes $[\mathbf{M}]$ with Stiefel number $w_{2} \cdot \bar{w}_{n-2}(M)=0$.

(ii) If $n \equiv 3$ (4) $I \Omega_{n, n-2}$ is isomorphic to $\Omega_{n} \oplus \mathbf{Z}_{2} \oplus \mathbf{Z}_{8}$ if $\alpha(n+1) \geqslant 3$ and to $\Omega_{n} \oplus \mathbf{Z}_{4}$ if $\alpha(n+1)=1$. If $\alpha(n+1)=2$ then $I \Omega_{n, n-2}$ is an extension of $\Omega_{n} \oplus \mathbf{Z}_{4}$ by $\mathbf{Z}_{2}$.

(iii) If $n \equiv 2$ (4) then $I \Omega_{n, n-2}$ is an extension of $\Omega_{n} \oplus \mathbf{Z}_{2} \oplus \mathbf{Z}_{2}$ by $\mathbf{Z}_{2}$.

(iv) If $n \equiv 1$ (4) then $I \Omega_{n, n-2}$ is an extension of $\Omega_{n} \oplus \mathbf{Z}_{2}$ by $\mathbf{Z}_{2}$.

Proof. Let $n \equiv 0(4), \alpha(n) \geqslant 2$. We have oommutative diagrams:

$$
\begin{aligned}
& 0 \rightarrow \mathbf{Z}_{2} \rightarrow I \Omega_{n, n-2} \rightarrow \Omega_{n} \rightarrow 0
\end{aligned}
$$

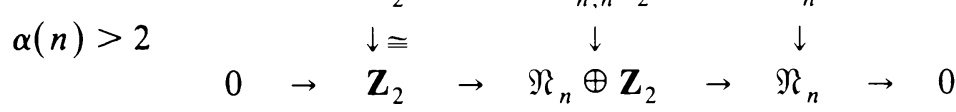

$$
\begin{aligned}
& \alpha(n)=2 \\
& 0 \rightarrow \mathbf{Z}_{2} \rightarrow I \Omega_{n, n-2} \rightarrow \Omega_{n} \rightarrow 0 \\
& 0 \rightarrow \mathbf{Z}_{2} \rightarrow \mathfrak{N}_{n / \mathbf{Z}_{2}} \oplus \mathbf{Z}_{4} \rightarrow \mathfrak{N}_{n} \rightarrow 0
\end{aligned}
$$

The upper diagram shows $I \Omega_{n, n-2} \cong \Omega_{n} \oplus \mathbf{Z}_{2}$ if $\alpha(n)>2$. If $n=2^{m}+2^{l}$ then the $\mathbf{Z}_{4}$ factor of $I \Re_{n, n-2}$ is generated by an immersion of $\mathbf{R} P^{2^{m}} \times \mathbf{R} P^{2^{l}}[\mathbf{K}]$. But $\mathbf{R} P^{2^{m}} \times \mathbf{R} P^{2^{l}}$ is not cobordant to an oriented manifold. Therefore the top sequence in the lower diagram also splits. 
If $\alpha(n)=1$ then the Dold manifold $P(1, n / 2)$ does not immerse up to cobordism in $\mathbf{R}^{2 n-1}$ as its number $\bar{w}_{2} \cdot \bar{w}_{n-1}$ is nonzero. Then (2.2) gives

$$
I \Omega_{n+1, n-2} \rightarrow \Omega_{n+1} \rightarrow \mathbf{Z}_{2} \stackrel{0}{\rightarrow} I \Omega_{n, n-2} \rightarrow \Omega_{n} \rightarrow \mathbf{Z}_{2} \rightarrow 0 .
$$

Now assume $n \equiv 3$ (4). If $\alpha(n+1) \geqslant 3$ we have a commutative diagram

$$
\begin{aligned}
& 0 \quad \rightarrow \quad \mathbf{Z}_{2} \oplus \mathbf{Z}_{8} \quad \rightarrow \quad I \Omega_{n, n-2} \quad \rightarrow \quad \Omega_{n} \rightarrow 0 \\
& \psi \downarrow \\
& 0 \quad \rightarrow \quad \mathbf{Z}_{2} \oplus \mathbf{Z}_{2} \oplus \mathbf{Z}_{2} \quad \rightarrow \quad \mathfrak{R}_{n} \oplus \mathbf{Z}_{2} \oplus \mathbf{Z}_{2} \oplus \mathbf{Z}_{2} \quad \rightarrow \quad \mathfrak{R}_{n} \quad \rightarrow \quad 0
\end{aligned}
$$

where $\psi(1,0)=(1,0,0)$ and $\psi(0,1)=(0,0,1)$. This implies that $I \Omega_{n, n-2} \cong \Omega_{n} \oplus \mathbf{Z}_{2}$ $\oplus \mathbf{Z}_{8}$. If $\alpha(n+1)=2$ then $\mathbf{C} P^{n+1 / 2}$ does not immerse up to oriented cobordism in $\mathbf{R}^{2 n-1}$ and coker $f: I \Omega_{n+1, n-2} \rightarrow \Omega_{n+1}$ is $\mathbf{Z}_{2}$. By (2.2) $\left|I \Omega_{n, n-2}\right|=8\left|\Omega_{n}\right|$ and sequence (2.3) reduces to

$$
0 \rightarrow \mathbf{Z}_{2} \rightarrow I \Omega_{n, n-2} \rightarrow \Omega_{n} \oplus \mathbf{Z}_{4} \rightarrow 0 .
$$

If $n+1$ is a power of 2 then $I \Omega_{n, n-2}$ fits into the exact sequence (2.3)

$$
\mathbf{Z}_{2} \oplus \mathbf{Z}_{2} \rightarrow I \Omega_{n, n-2} \rightarrow \Omega_{n} \oplus \mathbf{Z}_{2} \rightarrow 0
$$

and therefore has no elements of order 8. Sequence (2.2) takes the form

$$
I \Omega_{n+1, n-2} \stackrel{f}{\rightarrow} \Omega_{n+1} \stackrel{S}{\rightarrow} \mathbf{Z}_{2} \oplus \mathbf{Z}_{8} \rightarrow I \Omega_{n, n-2} \rightarrow \Omega_{n} \rightarrow 0
$$

where $S\left[\mathbf{C} P^{(n+1) / 2}\right]=(1,2) \in \mathbf{Z}_{2} \oplus \mathbf{Z}_{8}\left[\mathbf{0}\right.$, I.30]. It follows then that $I \Omega_{n, n-2} \cong \Omega_{n}$ $\oplus \mathbf{Z}_{4}$.

If $n \equiv 2$ (4) the result follows by comparing (2.2) with its unoriented analogue. The case $n \equiv 1$ is treated in the next section.

4. Double points and embeddings. The monomorphism $z(k) \rightarrow w(k)$ induces a homomorphism of the bordism groups $\Omega_{n-k}^{z(k)} \rightarrow \Omega_{n-k}^{w(k)}$. Moreover, there is a commutative diagram

$$
\begin{gathered}
\Omega_{n-k}^{z(k)} \rightarrow \Omega_{n-k}^{w(k)} \\
\partial \searrow \quad \swarrow D \\
I \Omega_{n, k}
\end{gathered}
$$

where $\partial$ and $D$ are described in $\S 2$.

4.1 Proposition. Let $k>2$. The natural homomorphism $\Omega_{i}^{z(k)} \rightarrow \Omega_{i}^{w(k)}$ fits into the following exact sequences:

$$
\begin{array}{lll}
0 \rightarrow \Omega_{1}^{f r} \rightarrow \Omega_{1}^{z(k)} \rightarrow \Omega_{1}^{w(k)} \rightarrow 0 & \text { for } k \text { even, } \\
0 \rightarrow \mathbf{Z}_{2} \oplus \mathbf{Z}_{2} \rightarrow \Omega_{2}^{z(k)} \rightarrow \Omega_{2}^{w(k)} \rightarrow 0 & \text { for } k \equiv 0(4), \\
0 \rightarrow \mathbf{Z}_{4} \rightarrow \Omega_{2}^{z(k)} \rightarrow \Omega_{2}^{w(k)} \rightarrow 0 & \text { for } k \equiv 1(4), \\
0 \rightarrow \mathbf{Z}_{2} \rightarrow \Omega_{2}^{z(k)} \rightarrow \Omega_{2}^{w(k)} \rightarrow 0 & \text { for } k \equiv 3(4) .
\end{array}
$$


The proof of 4.1 follows by comparing the corresponding long exact sequences of $[\mathbf{K}, 9.3]$. This result enables us to study the following diagram of exact sequences:

$$
\begin{array}{cc}
\Omega_{2}^{z(n-2)} & \Omega_{1}^{z(n-1)} \\
\partial \downarrow & \downarrow \\
\Omega_{n, n-2} \rightarrow I \Omega_{n, n-2} \rightarrow \Omega_{2}^{w(n-2)} \rightarrow E \Omega_{n-1, n-2} \rightarrow I \Omega_{n-1, n-2} \rightarrow \Omega_{1}^{n \cdot(n-1)} \rightarrow 0 \\
\downarrow & \downarrow \\
\Omega_{n} & \Omega_{n-1} \\
& \downarrow \\
& 0
\end{array}
$$

4.2 TheOREM. For $n>0, E \Omega_{n, n} \cong \Omega_{n}$.

4.3 THEOREM. Let $n>3$. If $n$ or $n+1$ is a power of 2 then $E \Omega_{n, n-1} \cong \Omega_{n}$; otherwise there is a short exact sequence

$$
0 \rightarrow \mathbf{Z}_{2} \rightarrow E \Omega_{n, n-1} \rightarrow \Omega_{n} \rightarrow 0 .
$$

This sequence splits if $n \equiv 2$ or 3 (4).

Theorem 4.2 follows immediately from sequence (2.4). Applying 4.1 to the previous diagram proves 4.3 for $n \equiv 0,2$ or 3 (4). If $n \equiv 1$ (4) we obtain the diagram

$$
\begin{aligned}
& 0 \\
& \downarrow \\
& \mathbf{Z}_{2} \oplus \mathbf{Z}_{2} \\
& \text { ว } \downarrow \\
& E \Omega_{n, n-2} \quad \rightarrow \quad I \Omega_{n, n-2} \stackrel{D}{\rightarrow} \quad \mathbf{Z}_{2} \oplus \mathbf{Z}_{2} \\
& \downarrow \nearrow \varphi \\
& \Omega_{n} \\
& \downarrow \\
& 0
\end{aligned}
$$

where the composite $\mathbf{Z}_{2} \oplus \mathbf{Z}_{2} \stackrel{\partial}{\rightarrow} I \Omega_{n, n-2} \stackrel{D}{\rightarrow} \mathbf{Z}_{2} \oplus \mathbf{Z}_{2}$ has kernel and cokernel $\mathbf{Z}_{2}$. This implies that $I \Omega_{n, n-2}$ is an extension of $\Omega_{n} \oplus \mathbf{Z}_{2}$ by $\mathbf{Z}_{2}$. We need to compute $\varphi$ above to know whether $D$ is onto. It is equivalent to study the problem of embedding oriented manifolds up to oriented cobordism in $\mathbf{R}^{2 n-2}$. Every orientable $m$-manifold embeds in $\mathbf{R}^{2 m-1}$ [Mas-P], thus we only need to investigate whether generators of $\Omega_{*}$ in dimension $n$ embed up to oriented cobordism in $\mathbf{R}^{2 n-2}, n \equiv 1$ (4). Using results of R. Brown [B, 2.1 and 5.1] and E. Thomas [Th, 1.1] it can be shown that all the $n$-dimensional generators of $\Omega_{*}$ given in [Wa] embed in $\mathbf{R}^{2 n-2}$ with the sole exception of the Dold manifolds $P\left(1,2^{l}\right), l>0$. Hence the double points homomorphism $D: I \Omega_{n, n-2} \rightarrow \mathbf{Z}_{2} \oplus \mathbf{Z}_{2}$ is onto if and only if $n-1$ is a power of 2 . This completes the proof of 4.3 .

4.4 CoRollary. If $M^{n}$ is orientable then $M$ embeds up to oriented cobordism in $\mathbf{R}^{2 n-2}$ if and only if the Stiefel number $w_{2} \cdot \bar{w}_{n-2}(M)=0$. If neither $n$ nor $n-1$ is a power of 2 this condition is always satisfied. 
D. Ellis [E] has recently proved 4.2-4.4 using different techniques. The following theorem extends one of his results.

4.5 THEOREM. If $k \equiv 0$ (4) there is short exact sequence

$$
0 \rightarrow \mathbf{Z}_{2} \oplus \mathbf{Z}_{2} \rightarrow E \Omega_{k+2, k} \rightarrow \Omega_{k+2} \rightarrow 0 .
$$

Proof. The exact sequences

$$
\begin{gathered}
E \Omega_{k+2, k} \rightarrow I \Omega_{k+2, k} \rightarrow \mathbf{Z}_{2} \rightarrow 0, \\
0 \rightarrow \mathbf{Z}_{2} \oplus \mathbf{Z}_{2} \oplus \mathbf{Z}_{2} \rightarrow I \Omega_{k+2, k} \rightarrow \Omega_{k+2} \rightarrow 0
\end{gathered}
$$

show that $\left|E \Omega_{k+2, k}\right| \geqslant 4\left|\Omega_{k+2}\right|$. On the other hand, Ellis [E] has proved that $E \Omega_{k+2, k}$ fits into the exact sequence

$$
E \Omega_{k+3, k} \rightarrow \Omega_{k+3} \stackrel{\partial}{\rightarrow} \mathbf{Z}_{2} \oplus \mathbf{Z}_{2} \rightarrow E \Omega_{k+2, k} \rightarrow \Omega_{k+2} \rightarrow 0,
$$

implying $\left|E \Omega_{k+2, k}\right| \leqslant 4\left|\Omega_{k+2}\right|$.

4.6 COROLlaRY. If $n \equiv 3$ (4) every oriented $n$-manifold embeds up to oriented cobordism in $\mathbf{R}^{2 n-3}$.

One final observation should perhaps be made. If $k \equiv 1$ (4) and $\Lambda$ denotes the kernel of $E \Omega_{k+2, k} \rightarrow \Omega_{k+2}$ then, as in 4.5, it can be shown that

$$
\Lambda= \begin{cases}\mathbf{Z}_{4} & \text { if } \alpha(k+3)>2, \\ \mathbf{Z}_{2} & \text { if } \alpha(k+3)=2 \\ 0 & \text { if } \alpha(k+3)=1\end{cases}
$$

Therefore, if $k+3$ is a power of 2 , then all codimension- $k$ isolated singularities in $2 k+3$ manifolds are orientably smoothable.

\section{REFERENCES}

[B] R. Brown, Immersions and embeddings up to cobordism, Canad. J. Math. 23 (1971), 1102-1115.

[C] R. Cohen, Immersions of manifolds, Proc. Nat. Acad. Sci. U.S.A. 79 (1982), 3390-3392.

[E] D. Ellis, Unstable bordism groups and isolated singularities, Trans. Amer. Math. Soc. 274 (1982), 695-708.

[K] U. Koschorke, Vector fields and other vector bundle morphisms, Lecture Notes in Math., vol. 847, Springer-Verlag, Berlin and New York, 1981.

[Mah-P] M. Mahowald and F. Peterson, Secondary cohomology operations on the Thom class, Topology 2 (1963), 367-377.

[Mas-P] W. Massey and F. Peterson, On the dual Stiefel-Whitney classes of a manifold, Bol. Soc. Mat. Mexicana (2) 8 (1963), 1-13.

[O] C. Olk, Immersionen von Mannigfaltigkeiten in euklidische Räume, Dissertation, Siegen, 1980.

[Sa] H. Salomonsen, On the homotopy groups of Thom complexes and unstable bordism, Aärhus Universitet, 1970.

[Sz] A. Szücs, Cobordism maps with simplest singularities, Lecture Notes in Math., vol. 788, SpringerVerlag, Berlin and New York, pp. 223-244.

[Th] E. Thomas, Embedding manifolds in Euclidean space, Osaka J. Math. 13 (1976), 163-186.

[Wa] C. T. C. Wall, Determination of the cobordism ring, Ann. of Math. (2) 72 (1960), 292-311.

[We] R. Wells, Cobordism groups of immersions, Topology 5 (1966), 281-294.

Departamento de Matemáticas, Centro de Investigación y de Estudios Avanzados del IPN, Apartado Postal 14-740, 07000, MExico, D. F. Mexico 